\title{
Philosophiques
}

\section{Le meilleur régime selon Leibniz}

\section{Jérémie Griard}

Volume 31, numéro 2, automne 2004

URI : https://id.erudit.org/iderudit/009811ar

DOI : https://doi.org/10.7202/009811ar

Aller au sommaire du numéro

\section{Éditeur(s)}

Société de philosophie du Québec

\section{ISSN}

0316-2923 (imprimé)

1492-1391 (numérique)

Découvrir la revue

Citer cet article

Griard, J. (2004). Le meilleur régime selon Leibniz. Philosophiques, 31(2),

349-372. https://doi.org/10.7202/009811ar

\section{Résumé de l'article}

Peu connue à cause de l'absence d'ouvrage de référence qui la présenterait dans toute son étendue, la pensée politique de Leibniz présente cependant un intérêt non négligeable pour l'histoire de la philosophie politique. En concevant l'homme comme sociable par nature, Leibniz ne fait pas pour autant de l'État l'aboutissement des sociétés naturelles, mais plutôt un moment passager dans la réalisation du monde moral au sein du monde naturel. Dès lors, le meilleur régime pour un tel État, ne résultant pas d'un contrat social bien que sujets et souverain y soient dans un rapport mutuel d'obligation, serait celui qui permettrait d'allier le mieux puissance et sagesse. Refusant tout pouvoir arbitraire, et insistant sur la nécessité de distinguer les pouvoirs, Leibniz considère la monarchie comme le meilleur régime en ce qu'elle serait plus facilement amendable : assisté d'un parlement, le puissant monarque ne pourrait qu'être sage.
Ce document est protégé par la loi sur le droit d'auteur. L'utilisation des services d'Érudit (y compris la reproduction) est assujettie à sa politique d'utilisation que vous pouvez consulter en ligne.

https://apropos.erudit.org/fr/usagers/politique-dutilisation/ 


\title{
Le meilleur régime selon Leibniz
}

\author{
JÉRÉMIE GRIARD \\ Université de Montréal \\ jeremie.griard@umontreal.ca
}

\begin{abstract}
RÉSUMÉ. - Peu connue à cause de l'absence d'ouvrage de référence qui la présenterait dans toute son étendue, la pensée politique de Leibniz présente cependant un intérêt non négligeable pour l'histoire de la philosophie politique. En concevant l'homme comme sociable par nature, Leibniz ne fait pas pour autant de l'État l'aboutissement des sociétés naturelles, mais plutôt un moment passager dans la réalisation du monde moral au sein du monde naturel. Dès lors, le meilleur régime pour un tel État, ne résultant pas d'un contrat social bien que sujets et souverain y soient dans un rapport mutuel d'obligation, serait celui qui permettrait d'allier le mieux puissance et sagesse. Refusant tout pouvoir arbitraire, et insistant sur la nécessité de distinguer les pouvoirs, Leibniz considère la monarchie comme le meilleur régime en ce qu'elle serait plus facilement amendable : assisté d'un parlement, le puissant monarque ne pourrait qu'être sage.
\end{abstract}

\begin{abstract}
Little-known because of the lack of a work of reference that would present it in all its extent, Leibniz's political thought is however of significant interest for the history of political philosophy. By conceiving humans has sociable by nature, Leibniz doesn't depict the State as the outcome of natural societies, but rather as a passing moment in the realization of the moral world within the natural world. Therefore, the best regime for such a State, not resulting from a social contract although subjects and sovereign are there in a mutual relationship of obligation, would be the one uniting best power and wisdom. Refusing every arbitrary power and emphasizing on the necessity of distinguishing powers, Leibniz considers monarchy as the best regime for it would be more easily improvable: assisted by a parliament, a potent monarch could only be wise'.
\end{abstract}

Question récurrente de la philosophie politique, la détermination du meilleur régime, c'est-à-dire du régime qui présenterait à la fois la plus grande viabilité et la plus grande justice possibles, n'a pourtant pas suscité chez Leibniz une réflexion particulière. Autant multiplie-t-il les projets de remise en ordre du corpus du droit, les plans concernant l'organisation économique de la société, ainsi que la création d'académies pour favoriser les découvertes dans tous les domaines, autant néglige-t-il de s'expliquer sur l'organisation politique d'un État².

Cependant, l'absence d'unité de développements explicitement consacrés à cette question ne doit pas laisser penser que de telles considérations lui sont cet article.

1. Nous tenons à remercier $\mathrm{M}^{\text {lle }}$ Sophie Lupien pour son assistance dans l'élaboration de

2. C'est d'ailleurs la raison du manque d'intérêt dont est victime la pensée politique de Leibniz de la part des historiens de la philosophie qui n'ont tenté, dans de très rares cas mentionnés à titre indicatif dans la bibliographie, que des approches synthétiques, jamais analytiques, de cette pensée. 
étrangères. Au contraire, leur éparpillement devrait inciter à retrouver, en filigrane, la position leibnizienne relative au meilleur régime ou encore, l'organisation politique qui répondrait le mieux au modèle qui pourrait se déduire de telles considérations.

Avant de tenter une confrontation entre les différents régimes et le modèle ainsi déduit, il est nécessaire pour cette déduction de présenter la genèse de l'État. Or la formation de l'État, et conséquemment de son régime, renvoyant à des conditions qui ne sont pas pour Leibniz universelles, la question du meilleur régime ne pourra être abordée - mais il eût été étonnant qu'il en fût différemment chez Leibniz - que comme celle du meilleur des régimes possibles.

\title{
La constitution naturelle de la société
}

Reprenant une thématique aristotélicienne, Leibniz fait de la créature une créature naturellement disposée à vivre en société, qu'elle soit humaine ou animale : " [L]a nature donne à l'homme et même à la plupart des animaux de l'affection et de la douceur pour ceux de leur espèce ${ }^{3}$.» Cet «instinct général de société, qui se peut appeler philanthropie dans l'homme ", conduit les hommes à s'unir "pour mieux obtenir leur but, comme les oiseaux s'attroupent pour mieux voyager en compagnie, et comme les castors se joignent par centaines pour faire des grandes digues, où un petit nombre de ces animaux ne pourraient réussir ${ }^{5}$ ».

Cette anthropologie leibnizienne, faisant de l'homme une créature sociable par nature, s'oppose à une théorie contractualiste déniant l'existence de toute société en dehors de l'État et défendant l'idée selon laquelle la société, qui se confond donc avec la cité, n'apparaît qu'à la faveur d'une situation intenable. Contre Hobbes, et avec Aristote, Leibniz accorde que l'homme est fait pour la société, et n'y est pas «seulement forcé par la nécessité et par la méchanceté de ceux de son espèce ${ }^{6}{ }$. Pas plus que chez les animaux, la société chez les hommes ne naîtrait par contrat.

La société et la cité étant distinguées, puisque «il est évident que toute Cité est une Société, mais non l'inverse ${ }^{7}$ », Leibniz efface le clivage entre l'état naturel et l'état civil puisque l'état naturel, compris comme absence d'état civil, c'est-à-dire de cité, d'État, peut cependant embrasser des sociétés naturelles et ne se limite pas à n'être qu'un état d'absence de société. S'il y a un instinct général de société, toutes les sociétés sont donc constituées par instinct. Or, si tel est le cas, l'universalité de la raison poussant à la constitution de sociétés, qui toutes sont naturelles, ne permet pas d'identifier une spéciation entre ces sociétés, les unes destinées à être des cités et les autres non. La constitution de sociétés est

\author{
3. Leibniz, Nouveaux essais, p. 73. \\ 4. Ibid. \\ 5. Ibid., p 213. \\ 6. Ibid. \\ 7. In Severinum de Monzambano, AK, IV, 1, p. 501.
}


nécessaire, mais d'une nécessité naturelle. C'est ainsi la raison pour laquelle Leibniz reprend à Aristote l'idée d'une continuité des différentes sociétés naturelles $^{8}$. Il n'y aurait donc rien dans ces sociétés qui permettrait de les distinguer entre elles quant à leur rapport à l'État, les unes étant pré-étatiques alors que les autres seraient non étatiques. Le passage déterminant pour l'apparition de l'État se ferait donc après la constitution de ces sociétés et ne se jouerait pas dès leur apparition. Cela signifierait-il que l'émergence de la cité est elle-même naturelle, et que l'État apparaîtrait nécessairement dans le prolongement des sociétés naturelles, Leibniz rejoignant Aristote également sur ce point? Les sociétés naturelles sont naturellement incluses les unes dans les autres, certes, mais cependant Leibniz rompt la continuité aristotélicienne en ce qui concerne la cité. En effet, les sociétés sauvages d'Amérique du Nord apportent l'exemple contraire. Il s'agit bien de sociétés, mais pas de cités. L'universalité du déploiement de cette continuité, tenue pour acquise à partir de l'Europe, est invalidée par cet exemple exotique :

Les Iroquois et les Hurons, Sauvages voisins de la Nouvelle-France et de la Nouvelle-Angleterre, ont renversé les Maximes politiques trop universelles d'Aristote et de Hobbes; ils ont montré par une conduite surprenante que des Peuples entiers peuvent être sans Magistrats et sans querelles, et que par conséquent les hommes ne sont ni assez portés par leur bon naturel, ni assez forcés par leur méchanceté à se pourvoir d'un gouvernement et à renoncer à leur liberté9

Leibniz s'appuie sur Aristote pour contredire Hobbes, puis sur un exemple pour contredire à son tour Aristote. "[L]es hommes ordinairement ne sont ni assez méchants ni assez bons; et Machiavel a bien remarqué que les deux extrémités sont également rares ${ }^{10}[\ldots]$. » Aristote et Hobbes s'opposent radicalement et Leibniz, quant à lui, s'oppose à ce couple dont chacun n'est que l'extrémité d'une universalité erronée. Dans ces conditions, il devient très difficile de rendre compte des raisons qui déterminent l'apparition de l’État. D'un point de vue naturel, celle-ci ne serait pas nécessaire mais contingente, à l'inverse de la constitution des sociétés. Comment, dès lors, justifier l'État?

\section{La constitution artificielle de l'État}

Les lois naturelles ne suffisent pas à rendre raison de la constitution de l'État si bien que ses lois fondamentales ne peuvent pas être assimilées à de simples lois naturelles, identiques à celles qui constituent les sociétés. La justification de l'État est donc à trouver en dehors de cette nécessité naturelle, en établissant la différence entre d'une part l'État, société en particulier, et d'autre part la société en général, tout en conservant le lien logique qui existe entre société

8. Cf. les remarques de Leibniz sur les Elementa juris universi et in specie publici Justinianaei de Felden, dans Ex Elementis Feldenii Excerpta et subinde immutata, AK, VI, 4, pp. 2906-2907.

9. A Coste, annexe à la lettre du 30 mai 1712, GP, III, p. 424.

10. Ibid. 
et État, pour ne pas faire de ce dernier une entité séparée de la société. L'État est une société, sans qu'on le confonde avec toutes les sociétés. Tout en étant différent, il lui ressemble.

La ressemblance entre société et cité repose sur leur finalité. Que la société soit ou non une cité, elle vise au bonheur de ses membres : «Ce n'est pas tant la nécessité, que l'inclination d'aller au meilleur, et d'approcher de la félicité, par l'assistance mutuelle qui fait le fondement des Sociétés et des États ${ }^{11}[\ldots]$..»

Toutefois la société naturelle, poursuivant le même but que la cité, se heurte à une limite, interdisant toute naturalité à l'État. Cette limite serait celle du lien social lui-même. L'instinct social porte sur le prochain, c'est-à-dire sur celui qui est à portée de sens grâce à sa proximité. Le lien social dans une société naturelle est concret. En revanche, dans l'État, le lien social, qui passe par le rôle joué par l'État, devient abstrait. Dans la société naturelle, chacun connaît les autres. Dans l'État, le lien social devient anonyme. Cet anonymat, cette abstraction du lien, permet de le démultiplier. Les hommes ne sont plus limités dans leur socialité par la proximité. Celle-ci peut se porter au-delà du prochain. En faisant de l'État une société naturelle, Aristote limitait le lien social à des rapports de proximité naturelle, condamnant l'État à ne pouvoir prendre que la forme la plus réduite qui soit, celle de la cité antique, société parfaite. Or, l'influence chrétienne conduit Leibniz à porter ses vues au-delà de cette cité, qui, n'étant qu'humaine, ne saurait offrir une perfection indépassable. La cité parfaite n'est pas ce petit État chez Leibniz, elle est la Cité universelle, celle de Dieu. Par conséquent, c'est la conception même de ce qu'est l'État qui s'en trouve changée. Pour Aristote il serait le plus petit possible, le plus grand pour Leibniz: "Ma définition de l'État ou de ce que chez les Latins est appelé Respublica est : que c'est une grande société dont le but est la sûreté commune ${ }^{12}$.» En demeurant naturelle, une société ne peut devenir grande. Or, si l'État est une grande société, il ne se constitue pas simplement de manière naturelle, mais de manière artificielle : les hommes dépassant ainsi leurs limites naturelles. En d'autres termes, la constitution d'une société permet aux hommes de dépasser leurs limites individuelles; la constitution d'un État leur permet de dépasser leurs limites mutuelles. Le mouvement va dans le sens d'un élargissement de la coopération, mais s'interrompt si l'on se cantonne à une simple naturalité. Une autre nécessité que la nécessité naturelle, ici impuissante, fait passer de la société naturelle à la société civile. Il s'agit d'une nécessité morale. Une société naturelle peut parfaitement répondre aux besoins naturels des hommes. C'est la raison pour laquelle une société dans laquelle le lien social ne serait que matériel se suffit à elle-même. Toutefois, une telle société se coupe de la réalisation du monde moral dans le monde naturel puisqu'elle se tient à l'écart de la réalisation de la République universelle, la Cité de Dieu. L'accès à l'universel exige en effet que soient dépassées les limites particulières.

11. Ibid.

12. A. Falaiseau, 8 juillet 1705, K, IX, p. 143. 
L'État se constitue donc, en tant que société, de manière naturelle, contrairement à l'idée défendue par Hobbes, mais, en tant qu'État, il se constitue de manière artificielle, par la recherche d'une socialité plus large, contrairement à celle défendue par Aristote. La constitution de l'État s'opère donc à la fois selon des lois naturelles et des lois morales, les premières renvoyant au monde créé par Dieu, les secondes à la République universelle dont Dieu serait le souverain. Cette Cité de Dieu, Leibniz la considère comme la plus parfaite et la plus haute des réalisations divines dans la mesure où s'y trouvent réunies et les lois naturelles et les lois morales. Pour autant, la morale n'est pas absente des sociétés sauvages dénuées d'État :

Cependant il faut avouer qu'il y a des points importants où les barbares nous passent, surtout à l'égard de la vigueur du corps, et à l'égard de l'âme même on peut dire qu'à certains égards leur morale pratique est meilleure que la nôtre, parce qu'ils n'ont point l'avarice d'amasser ni l'ambition de dominer ${ }^{13}$ (26, I, II, $\mathbb{2} 20$, p. 77).

Dans de telles sociétés, la morale y est en revanche plus pratique, c'està-dire plus tournée vers un intérêt immédiat. Les lois morales y sont comme subordonnées aux lois naturelles. En revanche, avec l'État, la transition s'opère de manière à ce que les lois morales dépassent les lois naturelles, la transition s'achevant dans la Cité de Dieu au sein de laquelle ce sont les lois naturelles qui se trouvent subordonnées aux lois morales.

On peut dire encore, que Dieu comme Architecte contente en tout Dieu, comme législateur; et qu'ainsi les péchés doivent porter leur peine avec eux par l'ordre de la nature; et en vertu même de la structure mécanique des choses; et que de même les belles actions s'attireront leurs récompenses par des voies machinales par rapport aux corps ${ }^{14}[\ldots]$.

D'une certaine manière, ces sociétés sauvages ne sont ni pires ni meilleures que les nôtres dans la mesure où «[i]l y a chez nous plus de bien et plus de mal que chez eux : un méchant Européen est plus méchant qu'un sauvage : il raffine sur le mal ${ }^{15}$ ». Leibniz aurait pu ajouter, en considérant l'autre versant du raffinement, qu'un bon Européen est meilleur qu'un sauvage, car, lui, raffinerait le bien. La différence entre sociétés sauvages, sans État, et sociétés civilisées, avec un État, serait dans la mesure et la démesure. Les dernières sont démesurées dans leur rapport au bien et au mal, la capacité à verser dans l'une des extrémités étant nécessairement accompagnée de celle de verser dans l'autre. En revanche, la méchanceté des sauvages est limitée du fait même de la limite à leur bonté. Il ne s'agirait donc pas de choisir entre l'une ou l'autre de ces sociétés, de préférer de moindres maux au détriment de plus grands biens, ou de plus grands biens au risque de subir de plus grands maux, mais de chercher une synthèse bénéfique, puisque, de l'avis de

13. Leibniz, Nonveaux essais sur l'entendement humain, p. 77.

14. Leibniz, La Monadologie, p 189.

15. Leibniz, Nouveaux essais sur l'entendement humain, p. 77. 
Leibniz, "rien n'empêcherait les hommes d'unir les avantages que la nature donne à ces peuples avec ceux que nous donne la raison ${ }^{16}$ ». Cette conciliation entre la nature et la raison est d'ailleurs ce dont il est question dans la Cité de Dieu. Dans le plan général de Dieu, les sociétés sauvages ne permettent pas, en elles-mêmes, d'élaborer cette Cité, pas plus que ne le permettraient les États eux-mêmes. La voie qui conduirait à la République universelle est une ligne de faite entre les deux versants de la condition humaine, à la fois être naturel et être rationnel, entre l'ubac que serait la société en tant que naturelle et l'adret que serait l'État. Puisque c'est l'équilibre qui importe, dans un cas comme dans l'autre, il est nécessaire de se tourner vers l'autre versant pour atteindre cette crête, c'est-à-dire pour les sociétés sauvages, de se tourner vers l'État pour ce qu'il a de rationnel, et pour les États, de se tourner vers les sociétés sauvages pour ce qu'elles ont de naturel.

Dès lors, la compréhension de la constitution de l'État ne peut se faire que par une double lecture, à la fois ex ante et ex post : ex ante, dans la mesure où l'État s'élabore par abstraction de la société pour en constituer une plus grande; $e x$ post, dans la mesure où cette constitution n'a de valeur qu'en tant que voie vers la Cité de Dieu qui exige que l'État se ressaisisse de ce qu'il peut y avoir de naturel. Le rapport entre raison et nature serait comme un rapport entre puissance et sagesse. Une société naturelle mais non rationnelle a une forme de sagesse, mais impuissante, tandis qu'une société rationnelle mais ayant perdu son rapport à la nature est puissante, mais d'une puissance qui peut s'exercer de manière déréglée autant vers le bien que vers le mal. C'est pourquoi les sauvages ne seraient, d'après Leibniz, ni pires ni meilleurs que les Européens, puisqu'ils n'auraient pas le pouvoir de l'être, et que les Européens seraient et pires et meilleurs qu'eux, puisqu'ils auraient le pouvoir de les surpasser en ce qui concerne les maux autant que les biens.

Pris dans une dynamique d'association des hommes entre eux pour garantir leur sécurité et obtenir leur bonheur, mouvement qui répond à la Gloire de Dieu, l'État ne serait pas le simple résultat définitif de son fondement. Comme la monade, il déploierait progressivement l'ensemble de ses virtualités, devenant toujours ce qu'il doit être. L'État se comprend donc luimême comme un moment passager entre deux états : d'une part, celui d'une simple coopération de proches entre eux; d'autre part, celui d'une communion de tous avec Dieu. Déterminé en amont par des raisons qui tiennent à la recherche d'une plus grande efficacité, l'État se détermine également en vue d'une fin morale à laquelle il doit aboutir. C'est la raison pour laquelle la forme qu'il prend doit répondre à des exigences relevant à la fois de la puissance et de la sagesse. Le meilleur État serait donc et sage et puissant, ce qui implique que l'organisation des pouvoirs en son sein permette l'union de ces deux qualités dans son régime. Mais puisque de la souveraineté dépend l'organisation des pouvoirs, il est nécessaire de la ressaisir dans son fondement avant de pouvoir trouver une organisation optimale du pouvoir.

16. Ibid. 


\section{Le fondement non contractuel de la souveraineté}

Comme nous l'avons vu à propos de l'origine de l'État, Leibniz s'oppose à la fois à Hobbes et à Aristote. De cette double opposition, il résulte que le rapport de Leibniz à un contractualisme étatique n'est pas univoque. En effet, si d'une part il rejette Hobbes et sa théorie contractualiste de l'origine de l'État comme passage de l'état de nature à un état civil, son rejet, d'autre part, d'une naturalité aristotélicienne de l'État, faisant par conséquent de la formation de celui-ci une constitution artificielle, soulève une équivocité quant à sa position vis-à-vis du contrat social.

Jusqu'à présent, il a surtout été question de sa défiance relative au contractualisme, afin d'insister sur les dispositions naturelles qui conduisent les hommes à constituer des sociétés, et qui s'accommodent donc difficilement du recours à cette sorte de compromis de chacun avec tous que serait le contrat. D'après Leibniz, les sociétés se constituent de manière naturelle. En revanche, les États se constitueraient de manière artificielle, ce qui permet ainsi de justifier l'absence d'équivalence entre État et société, puisque, selon lui, tout État est une société, mais non l'inverse. Les deux moments que nous mentionnions supra, celui de la société naturelle et celui de l'État, adjoints à cette équivocité du contrat social chez lui, pourraient évoquer une filiation entre Leibniz et Suárez.

Le jésuite espagnol semble lui aussi distinguer deux moments qui, pris ensemble, présentent une équivocité quant au contrat social. Selon lui, l'État en tant que tel serait une institution naturelle, tandis que sa forme, son régime, serait artificiel, si bien qu'originairement, la souveraineté résiderait dans l'ensemble de la communauté, tandis que ce ne serait que secondement que les hommes se livreraient à une quasi-aliénation de leur souveraineté au profit de celui qui deviendrait ainsi leur souverain : "Un tel transfert du pouvoir de la république au prince n'est pas une délégation, mais une quasi-aliénation, ou un abandon parfait du pouvoir qui était dans la communauté ${ }^{17}$. " Cette quasi-aliénation, qui est donc irrévocable contrairement à une délégation, et qui détermine le régime, est le résultat d'un arbitraire. Il y aurait ainsi naturalité de la souveraineté originaire, et artificialité de la souveraineté du prince. «Il est permis que ce pouvoir provienne absolument du droit de la nature, mais sa détermination à un certain mode de pouvoir et de régime résulte de l'arbitraire humain ${ }^{18}$.»

Contrairement à Hobbes, pour qui la désignation artificielle du souverain est l'acte par lequel se constitue organiquement l'État, Suárez, tout comme Leibniz, sépare la constitution de la communauté de la désignation du souverain. La première n'est pas contractuelle, mais naturelle, alors que la seconde n'est pas naturelle, mais contractuelle, par un contrat qui, pour sa part, évoque à son tour celui de Hobbes dans son irrévocabilité.

Cependant, bien que la comparaison entre Suárez et Leibniz soit plaisante, elle met surtout en évidence une différence qui permet de préciser l'opinion de

17. Suárez, De legibus, V, III, p. 186.

18. Ibid., p. 184. 
Leibniz. En effet, bien que la théorie suarézienne de l'origine de la souveraineté ne soit pas contractualiste, elle peut être considérée comme telle en ce qui concerne la désignation du souverain. Or, autant Leibniz se rapproche de lui pour ce qui est de la distinction entre l'État et ses institutions et une communauté préexistante, autant s'en éloigne-t-il pour ce qui serait de la désignation du souverain. La raison de cet éloignement est d'ailleurs l'argument leibnizien le plus fort à l'encontre des divers contractualismes. En effet, tous font de la souveraineté du souverain le fruit d'une convention. Serait souverain celui sur qui se serait porté le choix de ses futurs sujets. L'obligation de l'obéissance envers le souverain résulterait de sa désignation, la force obligative de la norme étant non dans son contenu mais dans sa forme, ou plus exactement dans la personne de celui qui la proclame. Or on sait avec quelles réticences Leibniz accueille cette position qui est celle de l'école moderne du droit naturel. Leibniz la rejette comme superflue dans la mesure où la fin, qui conduit elle-même à la constitution d'un État, suffit à faire du souverain le souverain, sans qu'il soit nécessaire de recourir à sa désignation conventionnelle. Toutes les théories contractualistes de l'État font de celui-ci le moyen par lequel les hommes obtiennent une sécurité et une félicité auxquelles ils ne pourraient prétendre en dehors. Dès lors, la désignation conventionnelle du souverain revient elle-même à désigner celui qui pourra le mieux garantir cette sécurité et cette félicité. Mais, et c'est en cela qu'intervient le reproche leibnizien, ne serait-ce que cette désignation qui ferait de celui sur qui se porte l'accord de tous le mieux à même d'assurer la fonction de souverain? Si seule la désignation était déterminante pour faire d'une personne le souverain, il n'y aurait donc de prime abord aucune raison suffisante pour justifier que le choix se portât sur telle personne plutôt que sur telle autre. Or, si un tel choix doit être opéré, il ne saurait porter lui-même que sur la personne qui serait la plus apte à être le souverain. Le souverain ne serait donc pas souverain en raison de la convention, mais en raison des qualités qu'il présente, justifiant par conséquent sa désignation, s'il y en avait une. La légitimité du souverain n'est pas formelle, elle est matérielle, à tel point qu'en commentant l'ouvrage de Sherlock, Leibniz, après avoir cité l'auteur, tire cette conséquence de la matérialité de la souveraineté :

Les sujets doivent maintenir le roi légitime contre les hommes, mais non contre Dieu. Question : si le prince usurpateur déclinait, en sorte qu'il était comme en balance avec celui qu'il a détrôné injustement, en sorte que les sujets pourraient aisément faire pencher la balance en faveur du légitime seigneur; savoir s'ils auraient droit de le faire... L'auteur croit que les pirates et voleurs n'ont pas leur autorité de Dieu. Mais je crois qu'entre un pirate et un concurrent injuste la différence n'est que du grand au petit, et qu'étant même soumis à un pirate je lui dois un certain degré de fidélité ${ }^{19}$.

19. Notes sur la dixième édition du livre de Sherlock, The Case of the Allegiance due to Soveraign Powers, G, pp. 887-888. 
Ainsi, comme Suárez, Leibniz défend, contre Hobbes, la distinction entre la société et l'État, mais, cette fois contre Suárez, il soulève l'inutilité d'une désignation conventionnelle du souverain. La souveraineté n'aurait chez lui aucun fondement contractuel, ni du point de vue de l'État, ni de celui du souverain. Et si Leibniz se réfère à un contrat social, ce n'est par conséquent que d'une manière très limitée : «il y a un quasi-contractus entre le gouvernement et celui qui jouit des avantages de la sûreté publique ${ }^{20}$.» Pour toutes les raisons que nous venons d'évoquer, il n'y aurait pas de contrat, mais seulement un quasi-contrat. Cette relativisation du propos met en évidence les bornes que Leibniz assigne au contrat. Celui-ci n'est considéré que dans ses effets, afin de décrire le mode selon lequel s'articulent les relations entre le souverain et ses sujets. La comparaison avec ce qui serait presque comme un contrat lui permet de souligner le rapport d'obligation réciproque entre le souverain et ses sujets : le premier devant garantir aux seconds sécurité et félicité; les seconds, pour autant que le premier respecte cette exigence, lui devant obéissance et fidélité. Toutefois, cette obligation réciproque ne naît pas d'un contrat, mais de la seule considération de l'utilité de ce rapport. Nous aurions comme les effets d'un contrat, sans pour autant qu'il y eût contrat.

Cette originalité donne la possibilité de ne retenir du contrat que ses effets les meilleurs. En effet, les théories contractualistes présentent deux faiblesses majeures, la seconde étant une tentative de réponse à la première. D'une part, si la souveraineté est fondée contractuellement, comme tout contrat, elle peut être dénoncée par l'une des parties, en l'occurrence les sujets soumis au souverain. Dès lors est ouverte la voie aux monarchomaques, pouvant arguer que le prince n'est souverain que tant que la communauté des sujets lui obéit, ces derniers pouvant, de manière tout aussi arbitraire que lorsqu'ils l'ont désigné, le déposer à tout moment. D'autre part, pour pallier cette instabilité et maintenir le contrat comme fondateur de la souveraineté, il faudrait poser un abandon par les sujets de tous leurs droits lors de la conclusion du contrat, et ainsi, afin de mettre à l'abri le souverain de toute subversion, rendre la souveraineté liberticide, liberté et sécurité étant considérées comme incompatibles pour les sujets.

Entre ces deux écueils d'un pouvoir absolu, qu'il s'agisse de celui des sujets ou de celui du souverain, Leibniz rétablit l'équilibre entre sujets et souverain en sapant les fondements contractualistes de la souveraineté, si bien qu'aux droits des uns ne correspondent plus simplement des obligations chez les autres, mais que, chez chacun, ses propres droits renvoient à ses propres devoirs.

\section{Les conséquences d'une souveraineté non contractuelle pour l'organisation des pouvoirs}

Dans la mesure où la souveraineté ne recevrait aucun fondement contractuel, aucune raison de principe ne jouerait en faveur d'une concentration de 
tous les pouvoirs entre les mains du seul souverain. Au contraire, puisque la souveraineté, qui ne se confond pas avec la majestée $e^{21}$, n'est pas un monopole de tous les pouvoirs mais, pour Leibniz, seulement de celui de la contrainte, du pouvoir d'exécution civile, il est nécessaire de ne plus parler de pouvoir mais de pouvoirs.

Cherchons à présent dans l'organisation des pouvoirs les indices qui pourraient militer en faveur d'un régime en particulier.

Nous avons vu précédemment de quelle façon Leibniz rejetait toute forme d'absolutisme, que celui-ci fût monarchique ou démocratique. Dans le commentaire qu'il donne en 1691 à propos de l'Avis important aux réfugiés de Bayle paru l'année précédente, il expose très clairement cette préférence pour la mixité des régimes :

Henning Arnisée, Allemand, et Alberico Gentile, Italien établi autrefois en Angleterre, ont écrit des livres exprès pour l'inviolabilité absolue ou, pour dire mieux, pour l'irrésistibilité des Souverains. D'autres qu'on appelle Monarchomaques, ont cru que la Souveraineté résidait proprement dans le peuple et que les princes ou les Assemblées suprêmes (tel qu'est le Grand Conseil de Venise) n'étaient que les premiers Magistrats. Ce Dogme se trouve enseigné par des docteurs de l'Église Romaine, aussi bien que par des auteurs réformés. Il n'y a que les universités de la Confession d'Augsbourg qui aient été exemptées de cette doctrine. Le parti le plus raisonnable a été pris de ceux qui ont jugé, avec Aristote, que le droit de souveraineté pouvait se trouver non seulement dans le peuple, mais encor dans un Prince ou dans un Grand Conseil ${ }^{22}[\ldots]$.

Cette inclination leibnizienne pour la mixité du régime place au second plan le régime lui-même, derrière la nécessité d'associer dans l'État la puissance et la sagesse, à tel point que Leibniz semble brouiller toutes les pistes qui permettraient d'identifier quel régime aurait sa préférence :

Quand on aime la véritable liberté, on n'est pas républicain pour cela, puisque la liberté raisonnable se trouve plus assurée lorsque le Roy et les assemblées sont liées par de bonnes lois, que lorsque le pouvoir arbitraire est dans le Roy ou dans la multitude ${ }^{23}$.

Tous les régimes en tant qu'ils prendraient une forme absolue sont rejetés et, inversement, tous sont acceptés pourvu seulement qu'ils évincent l'arbitraire, comme en témoignent ces différentes définitions :

Le but de la science politique à l'égard de la doctrine des Formes des Républiques, doit être de faire fleurir l'Empire de la raison. Le but de la Monarchie est de faire

21. «La Majesté est le droit de commander sans être commandé de qui que ce soit [...] la souveraineté est un pouvoir légitime de contraindre les sujets à obéir sans qu'on puisse être contraint soi-même, si ce n'est par une guerre. Ainsi la souveraineté serait le droit suprême et ordinaire d'exécution civile [...]. » Entretien de Philarète et d'Eugène touchant la souveraineté, AK, IV, 2, p. 308.

22. Sur l'Avis important aux réfugiés de Bayle, AK, IV, 4, p. 461.

23. A. Burnett, sans date, GP, III, p. 279. 
régner un héros d'une éminente sagesse et vertu, tel que votre Roi ${ }^{24}$ d'à présent. Le but de l'Aristocratie est de donner le gouvernement aux plus sages et aux plus experts. Le but de la démocratie, ou politie, est de faire convenir les peuples mêmes de ce qui est de leur bien. Et s'il y avait tout à la fois : un grand Héros, des Sénateurs très sages, et des citoyens très raisonnables, cela ferait le mélange des trois formes. Le pouvoir arbitraire est ce qui est directement opposé à l'Empire de la Raison ${ }^{25}$.

C'est sans doute la raison pour laquelle Leibniz s'est plus intéressé aux devoirs de l'État qu'à la forme que celui-ci devrait prendre, ses lois fondamentales étant indifférentes en ce qui les concernent. D’ailleurs, si «le but de la science politique » est «de faire fleurir l'Empire de la Raison », ce but ne peut pas être atteint par un et un seul régime en soi, tous pouvant être arbitraires, mais au contraire à partir de n'importe lequel d'entre eux. Un régime ne serait donc pas bon en raison de la seule nature de ses institutions. Ce ne sont pas les lois fondamentales qui importent, mais la finalité de l'État. Cellesci sont trop particulières et diverses pour faire l'objet d'une science en général, de la science politique en particulier :

Mais le Droit public dont vous parlez aussi est une chose trop variable, et les gens n'écoutent point raison là dessus. Il est tout autre en Angleterre et en Écosse qu'en Allemagne, et votre nation aussi bien que la nôtre se moquerait d'un Auteur qui voudrait raisonner sur les lois fondamentales ${ }^{26}$.

Puisque aucun régime ne se dégage nettement des autres comme étant le meilleur, rien ne servirait de subvertir l'un au profit d'un autre. Le déplacement de la problématique politique du choix du régime vers son mode de fonctionnement conduit Leibniz à adopter un conservatisme institutionnel, qui, pour autant, ne se satisfait pas des situations telles qu'elles lui sont contemporaines.

\section{La modération dans un conservatisme institutionnel}

Malgré les dysfonctionnements que peuvent rencontrer les régimes politiques, les cas pour lesquels il serait légitime de les renverser sont très rares :

Régulièrement et ordinairement les souverains doivent passer pour irrésistibles. C’est là le sentiment de feu M. Grotius, qui a été approuvé des personnes judicieuses. Cependant Monsieur Grotius lui-même a cru qu'il se peut trouver un cas extraordinaire où les inférieurs peuvent résister, c'est lorsqu'ils voient que les Supérieurs tendent manifestement à la destruction du peuple, ad eversionem salutis publicae; puisque le salut du peuple est la suprême loi. Mais la grande difficulté est de reconnaître ce cas. Et par conséquent on doit toujours être plus porté incomparablement à souffrir les caprices des Supérieurs qu'à

24. Guillaume III d'Angleterre.

25. Ibid., p. 277.

26. A. Burnett, 14 décembre 1705, ibid., p. 302. 
mettre tout en désordre pour leur résister; le remède ayant coutume d'être pire que le $\mathrm{mal}^{27}$.

Leibniz s'accorderait ici avec Robespierre, inspirateur de l'article 35 de la Déclaration des Droits de l'Homme et du Citoyen de la Constitution de l'an I, mais n'eût sans doute jamais accepté que soit érigé en une règle publique, à laquelle tous pussent recourir, ce principe : «Quand le gouvernement viole les droits du peuple, l'insurrection est, pour le peuple et pour chaque portion du peuple, le plus sacré des droits et le plus indispensable des devoirs. »

Leibniz marginalise à tel point le recours à la révolution qu'il semble s'accommoder de tous les régimes, aussi différents fussent-ils. Ainsi en va-t-il de ceux des Provinces-Unies ou de la Pologne. Au sujet du premier, Leibniz admet que Harrington dans son Oceana «a eu raison de recommander le gouvernement des Provinces-Unies, où on se range assez ordinairement à la raison dans les matières importantes de l'État ${ }^{28}$ ». Toutefois, le modèle ne serait pas exportable à la Pologne, à qui ce serait la monarchie élective, d'ailleurs le régime de la Pologne d'alors, qui conviendrait le mieux, comme en témoigne le début du Specimen demonstrationum pro eligendo rege Polonorum.

Leibniz passerait ici pour un flatteur si abstraction était faite des critiques qu'il sait également adresser à l'encontre des régimes, notamment des pamphlets qu'il dirige contre Louis XIV. Bien qu'il ne voie pas dans le roi de France un exemple hobbésien de l'absolutisme, puisque "une moitié de la France est constituée de Provinces qui sont dites les pays des Estats [...]; où le Roi ne peut assurément pas plus lever d'impôts extraordinaires, sans consultation des Ordres, que le Roi d'Angleterre dans son propre royaume ${ }^{29}$ ", ses tentatives répétées de réduire à néant des pouvoirs qui lui échappent suffisent à Leibniz pour brandir le spectre de l'absolutisme. La France n'a pas un régime absolu au dernier degré, mais que l'on prenne garde à ce qu'elle ne l'atteigne pas! Le retour au statu quo ante aura lieu dès la mort de Louis XIV, le Parlement recouvrant son pouvoir et cassant dès le lendemain de la mort du roi le testament par lequel il limitait les pouvoirs du régent Philippe d'Orléans pendant la minorité de Louis XV. Ni complètement imparfaits, ni complètement parfaits, tous les régimes sont susceptibles d'évolutions, et ce sont elles qui importent plus que le régime lui-même, afin que l'État demeure perfectible au lieu de sombrer dans une régression de ses qualités.

Leibniz place la recherche de la perfection dans la modération, modération des prétentions de chacun afin qu'elles ne fassent pas disparaître celles des autres. Il substitue, à l'alternative de la primauté souverain-sujets, l'alternative conciliation-opposition entre le souverain et les sujets. Il ne s'agit plus de savoir si le souverain prime sur les sujets, ou vice versa, s'il faut donner raison aux absolutistes monarchistes ou bien aux monarchomaques, mais au

27. Sur l'Avis important aux réfugiés de Bayle, AK, IV, 4, p. 461-462

28. A. Burnett, sans date, GP, III, p. 278.

29. De jure suprematus ac legationis principum Germaniae, AK, IV, 2, p. 59. 
contraire d'éviter toute opposition entre la finalité de la souveraineté et ce qui est poursuivi effectivement par le souverain. Le risque de distanciation est d'importance, que le souverain soit un monarque ou une assemblée, que cette dernière réunisse une partie des citoyens ou leur totalité. En effet, le souverain doit garantir la sécurité commune. Or «le Roi agit plus souvent en tant qu'homme qu'en tant que Roi ${ }^{30}$ ", laissant prévaloir des impressions personnelles sur des considérations universelles. La distanciation entre la fonction et la manière dont elle est remplie n'est pas le propre du monarque puisque le "pouvoir arbitraire peut se trouver non seulement dans les Rois, mais encore dans des assemblées, lorsque les cabales et les animosités y prévalent à la raison ${ }^{31}[\ldots]$ ». Si les buts de la monarchie, de l'aristocratie, et de la démocratie sont respectivement "de faire régner un héros d'une éminente sagesse et vertu ", "de donner le gouvernement aux plus sages et aux plus experts », « de faire convenir les peuples mêmes de ce qui est de leur bien ${ }^{32}$ », tous ceux qui accèdent au trône ne sont pas nécessairement ces héros, certains parmi ceux qui gouvernent peuvent être des intrigants, et le peuple lui-même peut se tromper ou être trompé. Pour réduire cet écart nuisible à la sécurité et à la félicité communes, c'est-à-dire pour garantir que le souverain soit en ce sens utile aux sujets, nul n'est besoin de changer de régime, ni même de bouleverser le régime lui-même. Il suffit d'en amender la pratique, en modérant les excès dont est susceptible le souverain. Plutôt que de s'en remettre aux aléas de la sagesse et de la vertu du souverain ou à ceux de ses capacités à écouter les meilleurs conseils des meilleurs conseillers, Leibniz préfère mettre l'accent sur un moyen plus sûr, par lequel le souverain serait lié au meilleur, sa volonté ne pouvant se porter que sur le meilleur pour tous.

Cette modération, qui est donc une limitation, n'est cependant pas une limitation du pouvoir du souverain. Il reste le détenteur du pouvoir coercitif dans toute son ampleur. Seulement, il serait souhaitable que soit limitée sa propre interprétation des motifs qui le conduisent à utiliser ce pouvoir coercitif. Ici se profile la distinction entre la majesté, droit de commander, et la souveraineté, droit de contraindre ${ }^{33}$. Bien qu'il soit théoriquement envisageable que le souverain réunisse puissance et sagesse, la distinction entre la majesté et la souveraineté se justifie par ses conséquences. En effet, la séparation de ces deux pouvoirs implique un équilibre de fait, divisant le risque de leur dévoiement. Par conséquent, la définition que Leibniz donne de la souveraineté, distincte de la majesté, préserverait de l'absolutisme et des risques qu'il comporte, et justifierait également le fait qu'il privilégie sa dimension externe par rapport à sa dimension interne dans les réflexions qu'il mène à son sujet, puisque, la souveraineté ne devant pas être confondue avec la majesté, et la

30. Codex juris gentium diplomaticus, D, IV, III, p. 288.

31. A. Burnett, sans date, GP, III, p. 277.

32. Ibid.

33. Cf. note 21. 
souveraineté étant le critère de l'existence d'un État, son concept s'ouvre sur un rapport à quelque chose qui serait en dehors de l'État, la majesté.

De plus, tout comme la souveraineté dans sa dimension interne préserve les droits de chacun, elle préserve, dans sa dimension externe, les particularismes. La multiplicité des pouvoirs, entendue par opposition à leur concentration, sauve à la fois l'unité de l'ensemble et sa cohérence, ainsi que les unités fondamentales et leur individualité. La souveraineté leibnizienne opère par une synthèse qui ne crée pas les particularismes, car dans ce cas il s'agirait d'une analyse réduisant le tout à ses parties, mais les sauvegarde, évitant ainsi de faire disparaître ces dernières au seul profit du premier : les parties sont conservées dans le tout, la diversité dans l'unité. Si la souveraineté, à l'interne, implique une analyse du pouvoir en des pouvoirs, à l'externe, elle implique leur synthèse. Le conservatisme se retrouve donc également à un niveau supraétatique, Leibniz ne cherchant qu'à articuler des institutions existantes, pour ainsi dire toujours déjà présentes.

L'exemple des entreprises leibniziennes en vue de l'obtention, par Hanovre, de l'électorat semblerait apporter un démenti à ce propos. Toutefois, force est de constater qu'il n'est pas question pour lui de bouleverser les institutions de l'Empire. En effet, la création d'un neuvième électorat ne remet en question ni l'Empire ni les autres Électeurs. Au contraire, dans la mesure où elle rééquilibre le rapport entre Électeurs catholiques et Électeurs protestants, où elle permet à la principauté de Hanovre de recevoir une distinction dont elle serait digne, comme Leibniz s'est efforcé de le montrer par ses différentes enquêtes, cette réforme pourrait être considérée comme conservatrice plutôt que révolutionnaire étant donné qu'elle renforcerait la cohérence de l'Empire au lieu de l'affaiblir. L'érection de la principauté de Hanovre en électorat est plus dans la continuité de l'Empire que dans une rupture avec celui-ci. Il en irait d'ailleurs ainsi de toutes les réformes, qui réformeraient l'institution existante pour lui faire retrouver sa forme pérenne plutôt que la lui faire perdre en la déformant. La politique leibnizienne n'est pas changement de direction, risque d'errance, mais accompagnement. C'est la raison pour laquelle Leibniz multiplie les conseils et non les injonctions, conseillant aux souverains d'être conseillés afin d'amender l'exercice du pouvoir. Le meilleur régime se comprend donc sous l'angle relatif de la convenance entre des moyens et des fins, les moyens et les circonstances étant les seuls éléments sur lesquels s'appuyer pour prétendre à ces fins, et non à remplacer, car de nouveaux moyens ne préjugeraient en rien de la réussite du projet.

N'étant pas justifiée formellement par un contrat social, la souveraineté ne tient sa légitimité, selon Leibniz, que de l'utilité qu'elle présente pour les sujets. Non contractuelle dans son fondement pour cette raison, elle peut néanmoins être considérée comme telle dans le rapport qu'elle doit faire naître entre le souverain et les sujets, de sorte que le premier comme les seconds se sentent réciproquement liés. En assignant à la souveraineté une source unique, les théories contractualistes ont tendance à concentrer tous les pouvoirs entre 
les mains d'un seul, le peuple ou le souverain. Or cette concentration, par l'absolutisme qui l'accompagne, va à l'encontre de la raison. Celle-ci commande en effet de fuir tout arbitraire, risque majeur que fait courir le souverain absolu auquel rien ne saurait ni ne pourrait s'opposer. Dans ces conditions, pour être plus conforme à la raison, l'organisation des pouvoirs devrait être une composition de différents pouvoirs plutôt qu'une concentration. Leibniz penchant en faveur de régimes mixtes, aucun des régimes que seraient la monarchie, l'aristocratie ou la démocratie, n'emporterait sa préférence. Il n'est pas question pour lui de choisir entre eux, ce qui supposerait que l'un serait meilleur que les autres, car aucun n'est meilleur ni pire que tous les autres. D'une certaine façon, tous se valent. La seule différence entre ses régimes est leur pratique. Il n'y aurait pour ainsi dire pas de meilleur régime, mais seulement de meilleures pratiques.

En raison de cette inversion du rapport, le régime ne déterminant pas la pratique, mais la pratique le régime, Leibniz peut accepter toutes les formes de régimes, tout en se permettant de critiquer, au cas par cas, leur pratique. C'est d'ailleurs la raison pour laquelle il ne se prononce pas plus explicitement en faveur de l'un plutôt que des autres, puisque tous les régimes se valent positivement sous leur rapport à la modération, et négativement sous celui de l'absolutisme et de l'arbitraire. L'équivalence des régimes implique un conservatisme institutionnel, soucieux de tenir compte de "ce qui se pratique aujourd'hui dans le monde ${ }^{34}$ ».

Toutefois, à défaut de ne retenir qu'un seul régime comme étant le meilleur, de manière absolue, il serait possible, malgré la relativité du meilleur régime, d'en retenir non pas plusieurs mais un seul, comme étant celui qui conviendrait le mieux et le plus souvent. Ce meilleur régime est donc largement dépendant d'évolutions historiques et de la situation contemporaine à Leibniz, comme nous allons l'aborder, après avoir successivement considéré les trois types de régimes, et leur degré de compatibilité avec l'ensemble des situations présentes dans l'Europe de la deuxième moitié du XVII ${ }^{\mathrm{e}}$ siècle.

\section{La souveraineté dans la pluralité : la démocratie}

Si la souveraineté réside dans plusieurs, alors il peut être question soit d'une démocratie, soit d'une aristocratie. Dans le premier cas, la collectivité est totale, l'ensemble de tous les citoyens étant souverain. Dans le second, la collectivité est limitée, n'étant souverain qu'un ensemble réduit aux seuls citoyens considérés comme les meilleurs. La différence entre les deux régimes repose sur une appréhension différente de la qualité des gouvernants. Pour ce qui est de la démocratie, tous peuvent également être amenés à gouverner, leur qualité abstraite de citoyens les rendant aptes de droit à l'exercice de la souveraineté. En revanche, pour ce qui est de l'aristocratie, ce sont les qualités individuelles, une aptitude de fait, qui prime sur l'aptitude de droit, la seconde ne pouvant être

34. Entretien de Philarète et d'Eugène touchant la souveraineté, AK, IV, 2, p. 290. 
conférée que par la première. Ces deux visions d'une souveraineté appartenant à la multitude s'opposent donc quant à ce rapport à l'aptitude à gouverner. Il est postulé soit que tous les hommes sont égaux, et qu'aucune discrimination n'est par conséquent nécessaire entre eux, soit le contraire.

Pour cette raison, l'anthropologie leibnizienne éloignerait de la démocratie. En effet, Leibniz soutient l'idée selon laquelle existeraient des inégalités de naissance entre les hommes.

[Ceux-ci naîtraient] avec des qualités différentes, les uns pour commander, et les autres pour obéir, afin que la puissance des souverains dans les monarchies et l'inégalité de ceux qui commandent et qui obéissent dans les Républiques, ne soient pas moins fondées sur la nature que sur la loi, et sur la vertu que sur la fortune ${ }^{35}[\ldots]$.

Cette justification d'une inégalité naturelle entre les hommes semble dictée par la nécessité de rendre compte d'inégalités constatées dans ce qui devraient être leurs effets. Dans tout État, qu'il s'agisse d'une monarchie ou d'une république au sens d'État dans lequel la souveraineté appartient à une assemblée, fût-elle celle de tout le peuple ou seulement des meilleurs, certains sont privés du gouvernement. Plutôt que de faire de cette différence entre les hommes le fruit d'un hasard, un effet sans cause, Leibniz préfère lui donner une certaine nécessité. Ainsi, ces différences de naissance substituent à la contingence de la loi ou de la fortune, la nécessité de la nature et de la vertu, les premières ne jouant plus qu'un rôle d'entérinement des secondes. Or, s'il est tenu pour assuré que les uns naissent pour commander, les autres pour obéir, il est difficile d'accepter un régime démocratique où tous seraient nés à la fois pour commander et obéir. Aussi, Leibniz, concevant la démocratie de manière classique et non encore moderne, limite son application aux seules cités exiguës telles qu'elles étaient déjà envisagées par Platon ou Aristote, y compris telles qu'elles le seront encore par Rousseau : "Cette forme démocratique ne convient qu'à de petits États, renfermés, pour ainsi dire, dans l'enceinte d'une cité, Athènes, Rome, Carthage, Syracuse ${ }^{36}$. »

Pourquoi limiter l'extension d'une démocratie? Justement à cause de l'absence de ce qui pourrait être appelé le postulat démocratique selon lequel les hommes seraient égaux de naissance. Puisque le gouvernement ne doit être confié qu'à ceux qui sont les plus aptes, et puisque les hommes n'ont pas tous cette aptitude à leur naissance, alors la seule situation dans laquelle tous pourraient de manière équivalente, voire de manière indifférente, être aptes à gouverner, ne pourrait être qu'une situation de base, n'exigeant qu'un minimum de compétence, minimum qui serait le plus petit dénominateur commun à tous. Or, une telle situation ne peut donc se trouver que dans des sociétés très petites. De telles sociétés présentent une complexité minimale

35. Portrait du prince tiré des qualités et des vertus hérö̈ques de S. A. $S^{m e} M^{g r}$. Jean Frédéric duc de Bronsvic et de Lunebourg, K, IV, p 461.

36. Specimen demonstrationum politicarum pro eligendo rege Polonorum, FC, VI, p 6. 
pour l'exercice de la souveraineté, non seulement dans sa dimension interne mais aussi dans sa dimension externe. Dans sa dimension interne, la souveraineté ayant pour fin d'assurer la sécurité et le bonheur commun, plus le nombre d'individus composant la société sera petit, plus l'intérêt commun sera proche de l'intérêt de chacun, et donc chacun serait apte à exercer la souveraineté. Dans sa dimension externe, c'est-à-dire en ce qui concerne l'exercice de la souveraineté en prenant part au droit des gens, la complexité est a fortiori minimale puisqu'un petit État n'est pas assez important pour se faire considérer à l'étranger par traités, armes et alliances, pour participer au droit des gens; donc il n'est besoin d'aucune qualité pour exercer la souveraineté externe puisqu'un petit État ne peut se prévaloir de cet exercice.

Ainsi la démocratie ne convenant qu'à de petits États, elle ne pourrait pas être le modèle le plus généralisable. Leibniz semble influencé par le déclin du modèle démocratique, disparu depuis l'Antiquité, s'étant effacé depuis le Moyen Âge devant l'aristocratie et la monarchie.

\section{La souveraineté dans la pluralité : l'aristocratie}

L'autre régime dans lequel la souveraineté appartient à une assemblée est celui de l'aristocratie, établi dans de nombreuses républiques, et, notamment, pour ne citer qu'une des plus prestigieuses d'entre elles, celle de Venise à laquelle Leibniz adressa d'ailleurs en 1713 sa Lettre d'un patriote. Dans ce texte, Leibniz vante les vertus de ce régime, par opposition à la monarchie.

Un prince est homme et mortel, les plaisirs l'occupent quelquefois plus que les affaires, il a ses caprices et ses passions et se laisse gouverner quelquefois par des ministres corrompus ou autres, de sorte qu'il peut arriver aisément qu'il néglige l'avenir pour jouir du présent. Mais la république est immortelle, un sénat est composé de plusieurs grands hommes zélés pour le bien de l'État, pour la gloire de la république et pour le salut de la postérité ${ }^{37}$.

L'aristocratie, gouvernement des meilleurs, serait-elle le régime qui conviendrait le mieux à l'ensemble des situations, des États, et ce, conformément à la conviction que Leibniz formule en ces termes : "Je crois que naturellement le gouvernement appartient aux meilleurs, comme il serait injuste que ceux qui n'entendent pas la marine voulussent être pilotes ${ }^{38}$ ?» Alors que la démocratie ne convient qu'à de petits États, l'aristocratie offre une extension plus large. La souveraineté n'y appartenant pas à tous, mais seulement à ceux qui sont les meilleurs, l'assemblée souveraine des aristocrates, quel que soit le nombre de personnes exclues de celle-ci, garantirait le bien commun, à la seule condition que les décisions prises par ce collège des sages le soient effectivement en suivant la raison. Cela pose donc ici le problème de la prise de décision par la pluralité.

37. Lettre d'un patriote à la Sérénissime République de Venise, FC, IV, pp. 177-178. 38. A. Burnett, 13/23 février 1700, GP, III, p. 271. 
En démocratie, puisqu'elle ne saurait être appliquée qu'à des nations exiguës, l'étroitesse du nombre de membres de la société permet une adéquation entre l'intérêt de chacun et l'intérêt commun. Or si l'évidence de cette adéquation décline proportionnellement au nombre de ces membres, dans une aristocratie, où certes la souveraineté appartient encore à une pluralité, mais une pluralité limitée inscrite dans un ensemble beaucoup plus vaste qui est celui de l'État, il n'est pas assuré que le bien commun coïncide exactement avec le bien particulier de chaque aristocrate, ni même que le bien commun des aristocrates ne soit pas séparé de celui de tous.

L'aristocratie est une forme d'État dans lequel le pouvoir est aux mains d'un collège des premières familles, c'est-à-dire de quelques citoyens. Il appartient donc aux plus puissants, qui ont un intérêt à maintenir un pouvoir fort. De là, des risques pour la liberté39 $[\ldots]$.

Le cas s'est d'ailleurs produit avec la République de Venise au sein de laquelle, à la suite de la conjuration de Tiepolo du 15 juin 1310, l'aristocratie, pour maintenir son pouvoir face au peuple, créa le Conseil des Dix, jouant le rôle de police politique et s'arrogeant progressivement tout le pouvoir exécutif qui jusqu'alors appartenait au Conseil des Sages composé de vingt-six membres nommés par cent vingt sénateurs élus par l'assemblée aristocratique qu'était le Grand Conseil.

Mais outre ces risques pour la liberté, la plus grande difficulté, faisant obstacle à l'adoption du modèle aristocratique comme le régime le plus généralisable, demeure la question de la délibération. Nécessaire pour faire surgir l'unité de la pluralité, le vote est essentiel pour tout régime dans lequel la souveraineté appartient à une assemblée. Il serait comme un pis-aller.

Le remède de la pluralité des voix données soit publiquement soit en secret, en ballottant, n'est pas suffisant pour réprimer ces abus. Les ballottations servent en quelque manière contre les cabales et font qu'il n'est pas difficile de s'assurer des suffrages par de mauvaises voies; mais elles ont cet inconvénient que chacun peut suivre son caprice et ses mauvais desseins, sans avoir la honte d'être découvert, et sans être obligé d'en rendre raison ${ }^{40}$.

La souveraineté étant le pouvoir exécutif, et demandant par conséquent une unité de l'exécution, elle ne peut être remise au sort, dans la mesure où celui-ci n'est pas infailliblement raisonnable. "Laissons aux théologiens le soin de discuter si le sort est un oracle du destin. Ces choses n'ont rien à voir avec la politique ${ }^{41}$.» Il ne reste donc que le vote, malgré toutes ses faiblesses. L'aristocratie ne serait donc pas totalement compatible avec la souveraineté leibnizienne. Celle-ci impose une unité qu'il est très difficile d'obtenir à partir d'une pluralité, puisque le moyen d'y parvenir, à savoir le vote, n'est qu'un expédient posant à son tour de nouvelles difficultés.

39. Specimen demonstrationum politicarum pro eligendo rege Polonorum, FC, VI, p. 6. 40. A. Burnett, sans date, GP, III, p. 277.

41. Specimen demonstrationum politicarum pro eligendo rege Polonorum, FC, VI, p. 7. 
Nous aurions chez Leibniz, sous le double rapport du rejet de l'arbitraire et de la recherche d'un équilibre au sein de l'État, comme une condamnation des régimes dans lesquels la souveraineté réside entre les mains de plusieurs. Si, contrairement à la monarchie, ils préviennent tout risque d'arbitraire d'un seul risque au contraire essentiel dans la monarchie, les moyens d'empêcher l'arbitraire de plusieurs sont insuffisants. Équilibrés en apparence, ces régimes courent le risque d'un absolutisme contre lequel il est difficile de combattre. En revanche, la monarchie, bien qu'elle ne soit pas équilibrée dans son fondement même, apparaît cependant plus amendable. Il serait en quelque sorte plus facile de faire participer plusieurs à l'exercice d'une souveraineté qui ne serait que dans un seul, que d'unifier son exercice tandis qu'elle serait dans plusieurs.

\section{La souveraineté dans l'unité : la monarchie}

Jusqu'à présent, nous n'avons procédé que de manière négative pour montrer quel pourrait être le régime le plus facilement compatible avec la majorité des États tels qu'ils se présentent dans l'Europe de la fin du XvII ${ }^{\mathrm{e}}$ siècle. Ni la démocratie, réservée à de petits États, ni l'aristocratie, dont les caractéristiques éloignent la pratique de la souveraineté de ce qu'elle devrait être, ne conviennent. Il ne resterait donc que la monarchie.

Puisque, dans son principe, la monarchie est le régime dans lequel la souveraineté n'appartient qu'à un seul, le monarque, l'unité que nécessite l'exercice de la souveraineté est obtenue par l'unité du souverain. Un seul est souverain et exerce la souveraineté. De cette manière se trouvent évités tous les problèmes liés à la façon de faire converger différentes volontés en une seule. La monarchie aurait pour elle la simplicité.

Cependant, cette simplicité ne préserve pas du risque d'arbitraire. Leibniz oppose également ce reproche à la démocratie et à l'aristocratie, dans la mesure où «ce pouvoir arbitraire peut se trouver non seulement dans les Rois, mais encor dans des assemblées ${ }^{42}$ ». Mais que ce qui peut être critiqué dans la monarchie puisse l'être aussi dans ces deux autres régimes ne sauve pas pour autant la première de cette dérive. C'est en cela que diffère principalement la monarchie de la démocratie et de l'aristocratie, et qu'elle recueille, nous semble-t-il, la préférence de Leibniz.

En effet, les régimes dans lesquels la souveraineté appartient à plusieurs échappent à l'arbitraire d'un seul. Mais comme l'arbitraire peut également être dans la multitude, ce point n'est d'aucune utilité. En revanche, même si la monarchie présente de prime abord un plus grand risque de dérive arbitraire, du fait que le souverain, étant unique, n'ait pas à composer dans l'État avec d'autres qui seraient ses pairs, elle offre néanmoins les conditions de possibilité d'une amélioration de l'exercice de la souveraineté. Des deux problèmes qui se posent aux régimes précédemment considérés, celui de l'efficacité d'une souveraineté qui doit composer un tout à partir de ses parties et celui de la fin

42. A. Burnett, sans date, GP, III, p. 277. 
qu'elle vise, à savoir le bonheur et la sécurité de tous, seul le dernier continue à se poser pour la monarchie. Le premier problème pourrait être considéré comme celui de l'efficacité de la souveraineté, et donc de sa forme, une forme unique étant préférable à une forme multiple; le second, quant à lui, comme celui de sa finalité, et donc de son contenu. Les deux problèmes nuisent réciproquement à leur résolution. D'une part, la forme de la souveraineté risque de dévoyer son contenu, et ainsi l'unité de la souveraineté risque de se faire au détriment de l'intérêt commun, comme dans le cas d'une entente entre aristocrates pour préserver leur souveraineté en limitant les libertés du peuple. D'autre part, le contenu de la souveraineté, quand bien même il serait juste ainsi que les décisions pour le bien commun, risque de ne demeurer qu'à l'état de virtualité, faute d'accord entre tous ceux qui forment le souverain.

Si le souverain n'est qu'un, toutes ses volontés seront exécutées, fussent-elles bonnes ou mauvaises pour l'intérêt commun. Inversement, si le souverain est composé de plusieurs, soit, faute d'accord, ses volontés, mauvaises mais aussi bonnes, ne sont pas exécutées, soit, un accord ayant été trouvé, elles le sont, mais alors bonnes comme mauvaises. L'efficacité d'un souverain monarque est supérieure à celle d'un souverain polyarque. D'ailleurs, Leibniz juge que «l'Empire ne peut être conservé que par les armes, qui demandent de la promptitude et un pouvoir absolu ${ }^{43}$ ». Cette défense d'un pouvoir unique fort, détenu par un seul, bien qu'elle réponde à un souci d'efficacité, ne suffit pas pour faire de toutes les monarchies en général, définies simplement comme un État dans lequel le souverain n'est qu'un seul et non une assemblée, le régime le plus convenable. Certes, la monarchie est le régime qui est le plus conforme à l'exercice de la souveraineté, mais cette conformité ne vaut comme telle que d'un point de vue pratique. Bien qu'il soit efficace, le moyen ne préjuge en rien de la fin. C'est pourquoi l'intérêt que Leibniz porte à ce régime se double de la recherche de solutions pour garantir que le pouvoir absolu nécessaire à la conservation de l'État ne devienne arbitraire.

Cette garantie, Leibniz la trouve, comme nous avons déjà eu l'occasion de le soulever, dans la participation au pouvoir d'entités distinctes du monarque, limitant non pas le pouvoir, mais son arbitraire. Au-delà de ce que R. Sève désigne comme une «monarchie limitée ${ }^{44}$ », il semble bien que ce soit plus précisément en faveur d'une monarchie parlementaire que se prononce Leibniz, c'est-à-dire d'une monarchie prenant en considération l'intérêt commun par le moyen de la représentation.

C'est un principe de politique qu'il y a d'autant plus d'ordre et de repos dans un État que la justice distributive y est mieux observée, dont les lois demandent entre autres, que plus les membres sont considérables, plus ils doivent prendre de part aux délibérations publiques. Autrement il se fait un divorce très préjudiciable à l'État entre la puissance de droit et celle de fait, si les suffrages ou

43. A. Ernest de Hesse-Rheinfels, 4/14 mars 1685, AK, I, 4, p. 351.

44. Sève, Leibniz : Le Droit de la raison, p. 57. 
voix de ceux qui sont puissants ne sont pas assez écoutés, et si ceux qui ont le plus de voix n'ont pas assez de puissance pour les appuyer. Cela fait que bien des résolutions sont sans effet, parce que ceux qui n'y ont pas eu grande part s'opposent à leur exécution, et ceux qui les ont formées sont obligés de recourir à des intrigues pour les faire réussir, ce qui se fait toujours aux dépens du bien public ${ }^{45}$.

Ainsi, il ne s'agit pas simplement de limiter le pouvoir du monarque mais de s'assurer que ceux qui sont les plus considérables y prennent part. In fine, la souveraineté n'appartient qu'au prince et non au peuple, mais son exercice ne saurait se réduire au seul souverain. En considérant le droit de souveraineté comme un droit qui s'accompagne nécessairement chez son titulaire de devoirs envers ceux sur lesquels il l'exerce, nous voyons comment le parlementarisme permet de faire fonctionner cette réciprocité entre le souverain et ses sujets. La réciprocité, pour être véritable, suppose une double indépendance des parlementaires à l'égard du souverain auprès duquel ils interviennent et de ceux dont ils sont les représentants, comme l'Angleterre en offre l'exemple :

Dans la Maison des communes du Parlement d'Angleterre, les Membres ne dépendent plus des Shires ou Bourgs qui les ont nommés, ne peuvent point être révoqués et ne doivent suivre que les mouvements de leur conscience ${ }^{46}[\ldots]$.

Dans une terminologie contemporaine, nous pourrions dire que, si mandat il y a, celui-ci est représentatif et non pas impératif. Ce caractère du mandat est justifié par deux raisons. D’une part, il ne peut être impératif, car, si tel était le cas, cela reviendrait à reconnaître une souveraineté populaire puisque, les électeurs pouvant démettre de leurs fonctions leurs représentants, ceux-ci auraient par conséquent l'obligation de porter leur volonté au niveau du pouvoir. Or Leibniz récuse toute souveraineté populaire pour les raisons que nous avons présentées à propos de la démocratie. D’autre part, le caractère simplement représentatif du mandat permet de faire participer les sujets au pouvoir, sans pour autant placer la souveraineté en eux. Dans les démocraties contemporaines, le mandat représentatif est fondé sur la représentation non pas du peuple dans son ensemble, mais sur celle de la nation, entité collective distincte du peuple. En procédant de la sorte, c'est-à-dire en faisant du mandat dont disposent les parlementaires un mandat représentatif et non impératif, Leibniz évince une souveraineté populaire, la souveraineté demeurant dans le souverain, mais parvient également à faire entrer la considération de l'intérêt commun dans l'exercice du pouvoir. Les représentants ne représenteraient pas le peuple souverain, mais la nation. La nation ainsi représentée n'est pas pour autant souveraine, dans la mesure où la souveraineté n'est

45. Écrit composé quelques mois avant la mort de feu Monseigneur l'Électeur Palatin touchant la création d'un neuvième Électorat en faveur des Protestants avec quelques Additions faites depuis, K, VI, p. 262.

46. «Observations sur le Projet de paix perpétuelle», Leibniz, Correspondance avec Castel de Saint Pierre, p. 39. 
pas exercée par cette assemblée représentative. En revanche, elle joue le rôle d'intermédiaire entre le souverain qu'est le monarque et ses sujets. Elle permet dès lors d'introduire auprès du souverain les exigences posées par l'intérêt commun, par la recherche de la sécurité et du bonheur de tous les membres de l'État, et non d'une fraction d'entre eux, sans que la souveraineté ne leur appartienne. Bien qu'elle en ait toutes les apparences, la monarchie parlementaire que prône Leibniz n'est pas une démocratie. Ni pure monarchie, ni pure démocratie ou aristocratie, elle est véritablement un régime mixte, participant des trois formes de gouvernement, que Leibniz compose par adjonction d'éléments disparates.

En effet, que le souverain ne soit qu'un, qu'il y ait monarchie et non pas polyarchie, ne signifie pas que le monarque gouverne absolument seul. Bien que la souveraineté n'appartienne qu'à lui, que les décisions ne relèvent en dernière instance que de sa personne, le souverain est entouré d'organes qui l'assistent, non qui le suppléent. C'est pourquoi le régime le plus convenable, s'accommodant le mieux avec la configuration générale des États qui sont dans leur majorité des monarchies héréditaires, est une monarchie dont la forme est amendée par l'existence d'un parlement, qui n'est pas souverain ni par conséquent un lieu de rapports de forces dans lequel serait en jeu le pouvoir lui-même. La modération se fait conciliation, toute voie médiane étant plus rationnelle qu'une voie extrême, comme l'expose Leibniz à Burnett dans une lettre du 30 octobre 1710 :

Il n'y a que les extrémités qui soient blâmables dans les Torries et dans les Whigs. Les modérés de part et d'autre s'accorderont aisément. Les extrêmes Torries sont les Jacobites, et les Whigs extrêmes sont les Républicains. Dites-moi un peu, Monsieur, si les Torries modérés ne reconnaissent point qu'il y a des cas extraordinaires où l'obéissance passive cesse, et où il est permis de résister au Souverain; et si les Whigs modérés ne demeurent pas d'accord qu'il ne vaut point venir légèrement ni autrement que pour des grandes raisons à cette résistance? Il en est de même du droit héréditaire de la succession, dont il ne faut point se départir, à moins que le salut de la patrie n'y force les peuples : car de croire qu'il y ait dans ces choses un droit divin indispensable, c'est aller jusqu'à la superstition, puisque cette rigueur n'a pas même lieu dans le Sabbat ni dans les autres préceptes divins positifs. Ainsi les gens raisonnables des deux partis n'étant pas fort éloignés dans l'essentiel, il me parait que toutes ces disputes ne sont que des amusements et des prétextes le plus souvent, et qu'on ne vise qu'aux charges et au pouvoir. Les Whigs ayant été les maitres durant quelques années ont rendu le gros de la nation jaloux d'eux et du Ministère. Si les Torries en usent de même, leur règne ne durera pas. Le meilleur serait d'employer de part et d'autre des gens capables et bien intentionnés, sans distinction de parti et d'opinion spéculative ${ }^{47}$.

Le gouvernement n'est pas affaire de rivalités, d'oppositions, de confrontations. Il est la recherche de l'équilibre permettant de servir l'intérêt public,

47. A. Burnett, 30 octobre 1710, GP, III, p. 320. 
le ménagement par le souverain des contraires dans le parlement et du parlement avec lui-même.

La réduction opérée par Leibniz de la souveraineté considérée simplement comme étant le monopole de la contrainte présente deux conséquences.

D'une part, il rend ainsi compte de toutes les situations politiques, quelle que soit la forme prise par le régime. En effet, quel que soit le régime, qu'il s'agisse d'une démocratie, d'une aristocratie ou d'une monarchie, le souverain est toujours celui qui dispose du pouvoir coercitif, le seul qui ait le droit de contraindre les sujets. Ce monopole de la contrainte est le dénominateur commun pour tous les souverains, y compris les souverains absolus qui cumulent tous les monopoles envisageables. En revanche, élargir la souveraineté audelà du monopole de contrainte conduit à nier l'existence de régimes modérés dans lesquels le souverain ne serait pas absolu. Parce que plus resserrée, la définition leibnizienne est universelle.

D'autre part, dans la mesure où la souveraineté n'est que le monopole de la contrainte, Leibniz peut récuser l'absolutisme à la fois comme n'étant pas nécessaire et même comme étant à éviter. Le monopole de la contrainte suffit à donner sa cohérence à l'État. Toutefois cette cohérence est plus ou moins facilement obtenue selon que le souverain est un ou multiple. Parce que la contrainte est un monopole, elle doit être unique. Or, pour qu'elle soit unique, il est nécessaire, si le souverain est constitué de plusieurs personnes, que ceuxci s'accordent entre eux. Mais la difficulté est telle qu'il serait préférable que le souverain ne soit qu'une seule personne, qu'il y ait monarchie plutôt que polyarchie. Si en tant que monopole de la contrainte, la souveraineté implique un monarque, parce qu'elle n'est que ce monopole-ci, la souveraineté exige également que le monarque ne soit pas le seul à prendre part au pouvoir. Les régimes qui font abstraction de la restriction de la définition sont des régimes absolus dans lesquels aucune limite ne s'oppose à l'arbitraire éventuel du souverain. Mais puisque le souverain n'est que celui qui a le droit de contraindre, afin d'offrir sécurité et bonheur à ses sujets, rien n'empêche ces derniers de participer au pouvoir. La représentation de l'intérêt commun, notamment par un parlement, permet un échange salutaire pour la paix civile et le bon fonctionnement de l'État. D'une part, en associant ceux qui en sont les plus dignes au pouvoir, le monarque n'est pas coupé de ses sujets, il peut ainsi connaître leurs aspirations. D'autre part, par ce moyen, il peut plus facilement s'assurer de leur soutien ou pour le moins désamorcer des incompréhensions à l'origine de mécontentements. La puissance du monarque n'est pas diminuée par ce dialogue, elle est rendue plus prudente, plus sage.

Ainsi, bien que Leibniz n'ait pas donné une formulation complète et explicite d'un régime qui serait le meilleur, ses conceptions politiques en dessinent un, adaptable aux États tels qu'ils sont, justifiant des monarchies parlementaires inspirées du modèle anglais, dans lesquelles le pouvoir n'est pas unilatéral, mais le résultat de l'harmonisation de plusieurs autour du souverain : Montesquieu ne publia De l'Esprit des Lois qu'en $1748 \ldots$ 


\section{Bibliographie}

AK : Leibniz. 1923-2... Sämtliche Schriften und Briefe, Berlin, AkademieVerlag.

D : Leibniz. 1768. Opera omnia, L. Dutens (dir. publ.), Genève, De Tournes.

FC : Leibniz. 1861-1875. CEuvres, L.A. Foucher de Careil (dir. publ.), Paris, F. Didot.

G : Leibniz. Textes inédits, G. Grua (dir. publ.), 1948, Paris, Presses Universitaires de France.

GP : Leibniz. 1875-1890, Die Philosophische Schriften, C.I. Gerhardt (dir. publ.), Berlin, Weidmannsche Buchhandlung.

$\mathrm{K}$ : Leibniz, Werke, O. Klopp (dir. publ.), Hanovre, Klindworth.

Leibniz. 1864-1884. Correspondance avec Castel de Saint Pierre, A. Robinet (dir. publ.), Paris, Thésaurus de philosophie du droit (Paris II C.N.R.S.-U.R.A. 956), 1995. . 1880. La Monadologie, E. Boutroux (dir. publ.), Paris, Delagrave. .1990. Nouveaux essais sur l'entendement humain, J. Brunschwig (dir. publ.), Paris, GF-Flammarion.

Naert, E. 1964. La pensée politique de Leibniz, Paris, Presses Universitaires de France.

Riley, P. 1972. The Political Writings of Leibniz, Cambridge, University Press. Robinet, A. 1994. G. W. Leibniz. Le meilleur des mondes par la balance de l'Europe, Paris, Presses Universitaires de France.

Sève, R. 1994. Leibniz, Le Droit de la raison, Paris, Vrin.

Suárez. 1856. De legibus, Paris, Vivès. 